\title{
Unstable Avoided Crossing in Coupled Spinor Condensates
}

\section{Citation}

Bernier, Nathan R., Emanuele G. Dalla Torre, and Eugene Demler. 2014. “Unstable Avoided Crossing in Coupled Spinor Condensates." Physical Review Letters 113 (6) (August). doi:10.1103/ physrevlett.113.065303.

\section{Published Version}

10.1103/PhysRevLett.113.065303

\section{Permanent link}

http://nrs.harvard.edu/urn-3:HUL.InstRepos:22856733

\section{Terms of Use}

This article was downloaded from Harvard University's DASH repository, and is made available under the terms and conditions applicable to Other Posted Material, as set forth at http:// nrs.harvard.edu/urn-3:HUL.InstRepos:dash.current.terms-of-use\#LAA

\section{Share Your Story}

The Harvard community has made this article openly available.

Please share how this access benefits you. Submit a story.

Accessibility 


\title{
Unstable Avoided Crossing in Coupled Spinor Condensates
}

\author{
Nathan R. Bernier, ${ }^{1,2, *}$ Emanuele G. Dalla Torre, ${ }^{3}$ and Eugene Demler ${ }^{3}$ \\ ${ }^{1}$ Department of Physics, Boston University, Boston, Massachusetts 02215, USA \\ ${ }^{2}$ Department of Physics, Ecole Polytechnique Fédérale de Lausanne (EPFL), 1015 Lausanne, Switzerland \\ ${ }^{3}$ Department of Physics, Harvard University, Cambridge, Massachusetts 20138, USA
}

(Received 4 March 2014; published 5 August 2014)

\begin{abstract}
We consider the dynamics of a Bose-Einstein condensate with two internal states, coupled through a coherent drive. We focus on a specific quench protocol, in which the sign of the coupling field is suddenly changed. At a mean-field level, the system is transferred from a minimum to a maximum of the coupling energy and can remain dynamically stable, in spite of the development of negative-frequency modes. In the presence of a nonzero detuning between the two states, the "charge" and "spin" modes couple, giving rise to an unstable avoided crossing. This phenomenon is generic to systems with two dispersing modes away from equilibrium and constitutes an example of class- $I_{o}$ nonequilibrium pattern formation in quantum systems.
\end{abstract}

DOI: 10.1103/PhysRevLett.113.065303

PACS numbers: 67.85.De, 03.75.Mn, 47.54.-r

Nonequilibrium conditions often lead to the spontaneous formations of regular patterns. This effect can be predicted by the appearance of dynamical instabilities at finite momentum, finite frequency, or both. For classical dissipative systems, several types of pattern formations were reviewed and classified by Cross and Hohenberg [1] (see Table I). With the advent of isolated quantum systems and, in particular, ultracold atoms, it is natural to inquire whether this phenomenon still occurs in the absence of a dissipative bath. Examples of pattern formation in unitary quantum systems were indeed observed in the roton softening of dipolar condensates [2] and in the dynamics of spinor condensates [3-7]. They can be described as mode-softening effects, in which the energy of a particular mode is reduced by changing the parameters of the system, until it reaches zero and the mode becomes unstable. This mechanism leads to unstable modes with finite momentum and vanishing real frequency and to the formation of a static pattern (class $I_{s}$ ).

In this Letter, we describe a generic mechanism for the formation of oscillatory patterns (class $I_{o}$ ) in unitary quantum systems, with a finite wave vector and a finite real frequency. As we explain in detail below, this type of instability occurs every time a dynamically stable mode crosses an energetically stable one (see Fig. 1). For example, this situation can be realized in tunneling-coupled condensates [8] and antiferromagnetic spinor condensates [9]. To observe it in a simple setting, we consider spinor condensates with an external coherent drive inducing Rabi oscillations between the states.

A system of this type was recently realized by Nicklas et al. [10], with two hyperfine states of ${ }^{87} \mathrm{Rb}$ coupled by a field close to atomic resonance. In this experiment, the initial state corresponded to a fully polarized condensate and the dynamics was induced by suddenly switching on the driving field. In contrast, we consider here the effects of a quench in the phase of the field. At a mean-field level, the system jumps from a minimum to a maximum of the energy and can remain dynamically stable. By considering the Bogoliubov excitations around this state [11], we find that instabilities necessarily arise around specific wave vectors, determined by an interplay between kinetic energy, coherent pump, and interactions.

To develop insight into the different types of pattern formation, we briefly review the stability conditions of quadratic modes in unitary quantum systems. Small fluctuations around a stationary state can be energetically stable, dynamically stable, or unstable. For a single degree of freedom, the energetically stable mode is illustrated by a

TABLE I. Classification of dynamical instabilities as static $(s)$ and oscillatory $(o)$, with examples of dissipative (classical) and unitary (quantum) systems. The focus of the present work is in the quantum domain of class $I_{o}$.

\begin{tabular}{|c|c|c|c|}
\hline Class & Dissipative & Unitary & Dispersion \\
\hline $\begin{array}{c}I_{s} \\
\left(k_{0} \neq 0,\right. \\
\left.\omega_{0}=0\right)\end{array}$ & $\begin{array}{l}\text { Eckhaus instability } \\
\text { in convection; } \\
\text { Turing } \\
\text { instability in } \\
\text { morphogenetics }\end{array}$ & $\begin{array}{l}\text { Mode softening } \\
\text { in } 2 \mathrm{D} \text { dipolar } \\
\text { condensates } \\
\text { and spinor } \\
\text { condensates }\end{array}$ & $\stackrel{\omega}{\uparrow}$ \\
\hline $\begin{array}{c}I I I_{o} \\
\left(k_{0}=0,\right. \\
\left.\omega_{0} \neq 0\right)\end{array}$ & $\begin{array}{l}\text { Belousov- } \\
\text { Zhabotinsky } \\
\text { chemical } \\
\text { reactions }\end{array}$ & $\begin{array}{l}\text { Pairing } \\
\text { instability } \\
\text { near } \\
\text { Feshbach } \\
\text { resonances }\end{array}$ & \\
\hline $\begin{array}{c}I_{o} \\
\left(k_{0} \neq 0,\right. \\
\left.\omega_{0} \neq 0\right)\end{array}$ & $\begin{array}{c}\text { Convection } \\
\text { in binary } \\
\text { mixtures }\end{array}$ & $\begin{array}{l}\text { Unstable } \\
\text { avoided } \\
\text { crossing in } \\
\text { spinor } \\
\text { condensates }\end{array}$ & $\omega$ \\
\hline
\end{tabular}



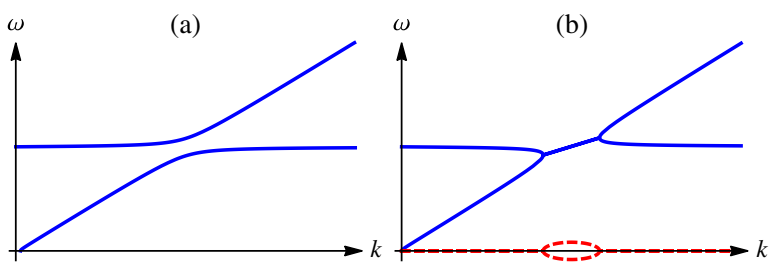

FIG. 1 (color online). A toy model illustration of the (a) stable and (b) unstable avoided level crossings. A mode with a linear dispersion $\left(\omega_{1} \propto k\right)$ is mixed with a mode of constant frequency $\left(\omega_{2}=\right.$ const $)$. In $(\mathrm{a})$, both modes are energetically stable $\left(\omega_{1}, \omega_{2}>0\right)$; in (b), the second mode is only dynamically stable $\left(\omega_{2}<0\right)$. The real component of the resulting eigenfrequencies is the solid blue line (positive by convention) and the imaginary component the dashed red line.

harmonic oscillator: $H=\frac{1}{2} \omega\left(p^{2}+x^{2}\right)$. The mode is characterized by a ground state and a real frequency $\omega$. In contrast, the dynamically stable mode is exemplified by $H=-\frac{1}{2} \omega\left(p^{2}+x^{2}\right)$. The dynamics are identical to the previous case (with the same frequency of oscillations), but all states now have negative energies and the system cannot represent thermal equilibrium. Finally, a mode can be dynamically unstable, illustrated by an inverted harmonic potential: $H=\frac{1}{2} \omega\left(p^{2}-x^{2}\right)$. Periodic oscillations are replaced by exponential growth, associated with an imaginary frequency $i \omega$ [12]. As demonstrated below, the notion generalizes to complex frequencies with real and imaginary components, implying both oscillatory and exponential dynamics.

We now consider the fluctuations of a manybody translation-invariant system. A collective mode is represented by a sum of harmonic oscillators $H=\sum_{k} \pm \frac{1}{2} \omega(k)\left(p_{-k} p_{k} \pm x_{-k} x_{k}\right)$, where the three stability scenarios are possible for each $k$. In particular, we consider two coupled modes described by

$$
H=\sum_{k} \frac{1}{2} \mathbf{p}^{\dagger} M_{p} \mathbf{p}+\frac{1}{2} \mathbf{x}^{\dagger} M_{x} \mathbf{x}
$$

with $M_{p}(k)$ and $M_{x}(k) 2 \times 2$ matrices, $\mathbf{p}=\left(p_{1, k}, p_{2, k}\right)^{\top}$, and $\mathbf{x}=\left(x_{1, k}, x_{2, k}\right)^{\top}$.

To diagonalize the system and find the Bogoliubov modes, we use a dynamical method similar to the formalism of Rossignoli and Kowalski [13]. We first compute the equations of motion of $x_{i, k}$ and $p_{i, k}$ in the Heisenberg picture

$$
\frac{d}{d t}\left(\begin{array}{l}
\mathbf{x} \\
\mathbf{p}
\end{array}\right)=\left(\begin{array}{cc}
\mathbb{D} & M_{p} \\
-M_{x} & \mathbb{1}
\end{array}\right)\left(\begin{array}{l}
\mathbf{x} \\
\mathbf{p}
\end{array}\right)
$$

We then define the Bogoliubov operators as the linear combination $b_{i, k}=a_{1} x_{1, k}+a_{2} x_{2, k}+a_{3} p_{1, k}+a_{4} p_{2, k}$ satisfying $i(d / d t) b_{i, k}=\omega_{i, k} b_{i, k}$. Going to the second time derivative, we finally obtain

$$
\mathbf{a}^{\top} \omega_{i, k}^{2}=\mathbf{a}^{\top}\left(\begin{array}{cc}
M_{p} M_{x} & \mathbb{1} \\
\mathbb{O} & M_{x} M_{p}
\end{array}\right)
$$

with $\mathbf{a}^{\top}=\left(a_{1}, a_{2}, a_{3}, a_{4}\right)$. The squared frequencies $\omega_{i, k}^{2}$ are given by the eigenvalues of $M_{x} M_{p}$ or $M_{p} M_{x}$.

Knowing how to diagonalize systems of the form (1), we introduce a simple model of level crossing with

$M_{p}=\left(\begin{array}{cc}\omega_{1}(k) & 0 \\ 0 & \omega_{2}(k)\end{array}\right), \quad M_{x}=\left(\begin{array}{cc}\omega_{1}(k) & \varepsilon \\ \varepsilon & \omega_{2}(k)\end{array}\right)$,

where $\varepsilon$ is some coupling introduced between the two modes. For $\varepsilon=0$, the two modes are decoupled and the eigenfrequencies of the system are simply $\omega_{1}(k)$ and $\omega_{2}(k)$. If $\omega_{i}(k)>0$, the mode is energetically stable, while if $\omega_{i}(k)<0$, it is only dynamically stable. For $\varepsilon \neq 0$, the original modes are mixed and we find the Bogoliubov (or eigen)frequencies by diagonalizing $M_{x} M_{p}$ for each wave vector $k$ :

$$
\omega^{2}(k)=\frac{\omega_{1}^{2}+\omega_{2}^{2}}{2} \pm \sqrt{\left(\frac{\omega_{1}^{2}-\omega_{2}^{2}}{2}\right)^{2}+\varepsilon^{2} \omega_{1} \omega_{2}} .
$$

The dynamics only depend on the squared eigenfrequencies, and by convention, we choose them to have positive real components, whichever the sign of $\omega_{1,2}$. At a level crossing, defined by $\left|\omega_{1}\right|=\left|\omega_{2}\right|$, the squared frequency difference is given by $\sqrt{\varepsilon^{2} \omega_{1} \omega_{2}}$. If both modes are energetically stable $\left(\omega_{1}, \omega_{2}>0\right)$, the difference is real, and one obtains the usual avoided level crossing [Fig. 1(a)]. In contrast, if one mode is energetically stable $\left(\omega_{i}>0\right)$ and the other only dynamically stable $\left(\omega_{j}<0\right)$, the frequency difference becomes imaginary and the avoided crossing unstable [Fig. 1(b)].

In this Letter, we consider an experimental system in which this unstable avoided crossing naturally arises: a condensate with two internal degrees of freedom [14-19] (states $|1\rangle$ and $|2\rangle$, separated by the energy $\hbar \omega$ ) coherently coupled by a radio-frequency (rf) field [20]. The rf coupling contribution to the Hamiltonian is $H_{\mathrm{rf}}=-\alpha\left(e^{i \nu t} \hat{\psi}_{1}^{\dagger} \hat{\psi}_{2}+e^{-i \nu t} \hat{\psi}_{2}^{\dagger} \hat{\psi}_{1}\right)$, with $\alpha>0$ the Rabi frequency, $\nu$ the frequency of the field, and $\hat{\psi}_{1}^{\dagger}$ and $\hat{\psi}_{2}^{\dagger}$ bosonic creation operators for states $|1\rangle$ and $|2\rangle$. In the interaction frame with respect to $H_{0}=\hbar \nu\left(\hat{\psi}_{2}^{\dagger} \hat{\psi}_{2}-\hat{\psi}_{1}^{\dagger} \hat{\psi}_{1}\right)$, time dependence is canceled and the Hamiltonian becomes

$$
\begin{aligned}
\hat{H}= & \int d^{d} x \sum_{i}-\hat{\psi}_{i}^{\dagger} \frac{\nabla^{2}}{2 m} \hat{\psi}_{i}+\frac{\lambda_{i}}{2} \hat{\psi}_{i}^{\dagger} \hat{\psi}_{i}^{\dagger} \hat{\psi}_{i} \hat{\psi}_{i}+\lambda_{12} \hat{\psi}_{1}^{\dagger} \hat{\psi}_{2}^{\dagger} \hat{\psi}_{2} \hat{\psi}_{1} \\
& +\frac{\delta}{2}\left(\hat{\psi}_{2}^{\dagger} \hat{\psi}_{2}-\hat{\psi}_{1}^{\dagger} \hat{\psi}_{1}\right)-\alpha\left(\hat{\psi}_{1}^{\dagger} \hat{\psi}_{2}+\hat{\psi}_{2}^{\dagger} \hat{\psi}_{1}\right)
\end{aligned}
$$

where we set $\hbar=1, m$ is the mass of the atoms, $\delta=\omega-\nu$ is the detuning, and the dimension $d=1,2,3$. The parameters $\lambda_{1}, \lambda_{2}$, and $\lambda_{12}$ describe state-dependent local interactions, 
which can be tuned with the aid of a Feshbach resonance (see Ref. [21] for a review).

The equilibrium properties of the Hamiltonian (6) are well understood [11]. In the absence of the coherent drive $(\alpha=0)$, the population of each state is fixed and the system undergoes a miscible-immiscible phase transition, depending on the relative strength of the interactions [22]. If $\left|\lambda_{12}\right|<\sqrt{\lambda_{1} \lambda_{2}}$, the gas is miscible; i.e., it is energetically favorable to have both species share space. Alternatively, if $\lambda_{12}>\sqrt{\lambda_{1} \lambda_{2}}$, the gas is immiscible: the phase-separated state has the lowest energy. Finally, if $\lambda_{12}<-\sqrt{\lambda_{1} \lambda_{2}}$, the system collapses. We note that phase transitions are signaled by dynamical instabilities. For example, immiscibility is triggered by waves of population imbalance ("spin mode") becoming unstable at long wavelength and pushing the gas towards the phase-separated state.

In the presence of a coherent drive, the mean-field phase diagram of the Hamiltonian (6) can be found by replacing the field operator by a classical field $\hat{\psi}_{i} \approx \Psi_{i}=\sqrt{n_{i}} e^{-i \theta_{i}}$ and looking for stationary solutions $\Psi_{i}(t)=\Psi_{i}(0) e^{-i \mu t}$ to the Gross-Pitaevskii equations [17]. This approach leads to two main families of solutions, characterized by the phase difference $\theta=\theta_{1}-\theta_{2}$ : when $\theta=0$, the coupling energy is minimal, and when $\theta=\pi$, it is maximal. For each value of $\theta$, there can be up to three stationary solutions corresponding to different values of the population imbalance $n_{1}-n_{2}$ [23]. Here, we consider only the symmetric solution $n_{1}=n_{2}$, which leads to simpler analytical results and can be prepared experimentally.

To study the stability of the mean field, we introduce small quantum fluctuations, approximating $\hat{\psi}_{i} \approx \Psi_{i}+$ $e^{-i \theta_{i}} \sum_{k \neq 0}\left(e^{i k x} / \sqrt{V}\right) a_{i, k}$ in Eq. (6) and expanding up to second order. For stationary mean fields, the linear term cancels and we are left with a quadratic bosonic system [24]. For convenience, we introduce the canonically conjugated variables $x_{i, k}=\left(a_{i,-k}^{\dagger}-a_{i, k}\right) / i \sqrt{2}$ and $p_{i, k}=\left(a_{i,-k}^{\dagger}+a_{i, k}\right) / \sqrt{2}$, which are, respectively, linked to phase and density fluctuations [25]. In this basis, the Hamiltonian takes the form (1) with the matrices $M_{p}$ and $M_{x}$ given by

$$
\begin{aligned}
M_{p}= & 2\left(\begin{array}{cc}
\lambda_{1} n_{1} & \lambda_{12} \sqrt{n_{1} n_{2}} \\
\lambda_{12} \sqrt{n_{1} n_{2}} & \lambda_{2} n_{2}
\end{array}\right)+\alpha \cos \theta\left(\begin{array}{cc}
\sqrt{\frac{n_{2}}{n_{1}}} & -1 \\
-1 & \sqrt{\frac{n_{1}}{n_{2}}}
\end{array}\right) \\
& +\frac{k^{2}}{2 m}\left(\begin{array}{ll}
1 & 0 \\
0 & 1
\end{array}\right)+\frac{\delta}{2}\left(\begin{array}{cc}
-1 & 0 \\
0 & 1
\end{array}\right), \\
M_{x}= & \alpha \cos \theta\left(\begin{array}{cc}
\sqrt{\frac{n_{2}}{n_{1}}} & -1 \\
-1 & \sqrt{\frac{n_{1}}{n_{2}}}
\end{array}\right)+\frac{k^{2}}{2 m}\left(\begin{array}{ll}
1 & 0 \\
0 & 1
\end{array}\right)+\frac{\delta}{2}\left(\begin{array}{cc}
-1 & 0 \\
0 & 1
\end{array}\right),
\end{aligned}
$$

and we can use Eq. (3) to find the eigenmodes.
We first consider the symmetric case $\lambda=\lambda_{1}=\lambda_{2}$ and $\delta=0$, with the mean-field solutions $n_{1}=n_{2}$ and $\theta=0, \pi$. In this case, $M_{x}$ and $M_{p}$ have the same eigenvectors for all $k$ and the system decouples into a charge mode $\left(\sqrt{2} x_{c}=x_{1}+x_{2}, \sqrt{2} p_{c}=p_{1}+p_{2}\right)$ and a spin mode $\left(\sqrt{2} x_{s}=x_{1}-x_{2}, \quad \sqrt{2} p_{s}=p_{1}-p_{2}\right)$. For the charge mode, the Hamiltonian is

$$
H_{c}=\frac{1}{2}\left[\left(n\left(\lambda+\lambda_{12}\right)+\frac{k^{2}}{2 m}\right) p_{c}^{2}+\left(\frac{k^{2}}{2 m}\right) x_{c}^{2}\right]
$$

which does not depend on the coupling $\alpha$ or the phase $\theta$. The condition for stability is that both terms are positive, or equivalently, $\lambda_{12}>-\lambda$. For the spin mode, we obtain

$$
\begin{aligned}
H_{s}= & \frac{1}{2}\left[\left(n\left(\lambda-\lambda_{12}\right)+2 \alpha \cos \theta+\frac{k^{2}}{2 m}\right) p_{s}^{2}\right. \\
& \left.+\left(2 \alpha \cos \theta+\frac{k^{2}}{2 m}\right) x_{s}^{2}\right],
\end{aligned}
$$

and both the coupling strength $\alpha$ and the phase $\theta$ will affect the stability of the system. For $\alpha=0$, the stability condition reduces to the miscibility condition $\lambda_{12}<\lambda$. For $\alpha \neq 0$ and the equilibrium solution $\theta=0$, this condition is simply extended to $\lambda_{12}<\lambda+2 \alpha / n$ [see Fig. 2(a)].

Things get more interesting for the nonequilibrium solution $\theta=\pi$. In this case, the coefficient in front of $x_{s}^{2}$ in Eq. (9) is always negative for small enough $k$. The
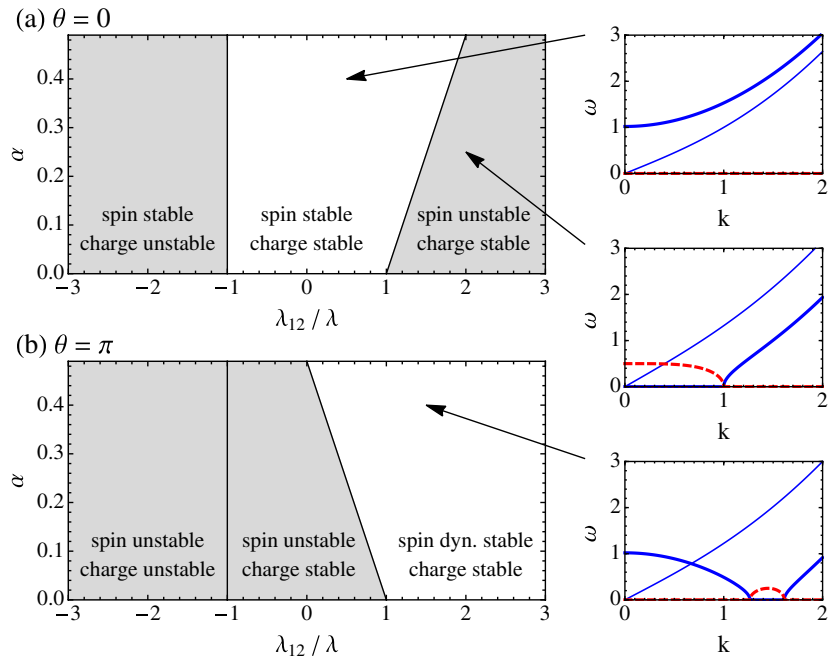

FIG. 2 (color online). Stability diagrams of the system for the symmetric case $\left(\lambda=\lambda_{1}=\lambda_{2}, n_{1}=n_{2}, \delta=0\right)$, with solutions (a) $\theta=0$ (equilibrium) and (b) $\theta=\pi$ (nonequilibrium). The grey background indicates dynamical instability at small wave vectors. The insets illustrate the real (solid blue line) and imaginary (dashed red line) parts of the dispersions of the two modes (thinner lines for the charge mode and thicker for the spin mode). The variables are in dimensionless units with $m=1$ and $\hbar=1$, and the energy is measured in $\lambda n$. 
system can nevertheless become dynamically stable when the coefficient in front of $p_{s}^{2}$ is negative as well, translating to the condition $\lambda_{12}>\lambda-2 \alpha / n$ [see Fig. 2(b)]. This is the result of a competition between the interactions and the coherent coupling, compensating each other to avoid the instability. For larger wave vectors, the kinetic energy $k^{2} /(2 m)$ will increase both coefficients until they switch sign. Except for the special case $\lambda_{12}=\lambda$ (where they are equal), there will be an unstable interval when one coefficient is positive and the other negative. This dynamical instability is of type $I_{s}$, not directly related to mode softening, and is shown in Fig. 2 (third inset).

If we depart from the symmetric case, by considering $\lambda_{1} \neq \lambda_{2}$ or $\delta \neq 0$, the matrices $M_{x}$ and $M_{p}$ will generally no longer have the same eigenvectors and the two modes will mix. For a small perturbation, the dispersions will be qualitatively affected only at energy crossings. This occurs for the nonequilibrium solution $\theta=\pi$, where the spin mode, first decreasing in frequency, crosses the charge mode, as shown in the third inset of Fig. 2. As explained above, a novel instability arises as the crossing between the energetically stable charge mode and the dynamically stable spin mode is avoided [see Fig. 3(a)]. Up to the first order in the perturbation $\lambda_{1}-\lambda_{2}$ or $\delta$, the instability is centered around

$$
k_{0}=\sqrt{2 m \alpha \frac{2 \alpha+\left(\lambda_{12}-\lambda\right) n}{2 \alpha+\lambda_{12} n}} .
$$

The nontrivial dependency on the coupling and interaction parameters signals that the instability results from a competition between the two.

We now describe an experimental protocol designed to probe the interesting properties of the $\theta=\pi$ solution and its instabilities. In order to achieve the relevant state, we propose to apply a finite coherent drive $\alpha$ and a large detuning $\delta$, and start with the atoms in the ground state $|1\rangle$.
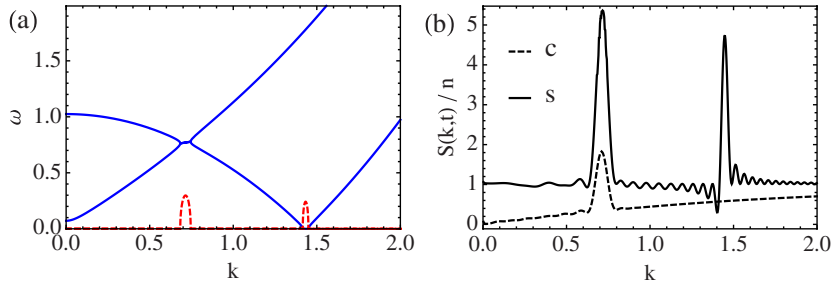

FIG. 3 (color online). (a) Dispersion relation in the asymmetric case $\delta \neq 0$, showing two dynamical instabilities (see the text). The real part of the frequency is the solid blue line, and the imaginary part (multiplied by 10 for better legibility) is the dashed red line. (b) Power spectrum of the charge (dashed line) and spin (solid line) sectors, showing sharp peaks in Fourier space where the dispersion relation displays instabilities. Numerical parameters: $\lambda_{12}=1.05 \lambda, \quad \alpha=0.5 \lambda n, \quad \delta=0.1 \lambda n$, and $t=40.0 \hbar / \lambda n$. The variables are in dimensionless units with $m=1$ and $\hbar=1$, and the energy is measured in $\lambda n$.
If the detuning $\delta$ is adiabatically reduced to a small finite value, the system will end up in the minimal-energy solution $\theta=0$. We then propose to quench the system by changing $\alpha \rightarrow-\alpha$ (by introducing a phase shift of $\pi$ in the $\mathrm{rf}$ field), equivalent to changing $\theta=0 \rightarrow \pi$. The system is now in the appropriate mean-field state $\left(\theta=\pi, n_{1} \approx n_{2}\right)$ that we let evolve for a time $t$ before proceeding to a measurement.

Because of quantum fluctuations, the unstable modes of the quenched Hamiltonian will develop and grow exponentially. Charge and spin density waves will form at the unstable wave vectors, creating regular patterns at the associated wavelengths. Information about those patterns can be found in the power spectra of the spin $(s)$ and charge (c) density fluctuations. Within the approximations of the present analysis, they are simply given by $S_{s / c}(k, t)=$ $2 n\left\langle p_{s / c}(k, t) p_{s / c}(-k, t)\right\rangle$ and plotted in Fig. 3(b). As expected, both $S_{c}(k, t)$ and $S_{s}(k, t)$ show sharp features at the wave vectors corresponding to the dynamical instabilities predicted by the dispersion relation shown in Fig. 3(a). In particular, the charge mode displays only one dynamical instability at $k \approx 0.7$, induced by the unstable avoided crossing with the (otherwise dynamically stable) spin mode [26].

In summary, new phenomena occur in the intermediary regime of coherently driven spinor condensates, where the coupling strength is comparable to the interaction energy. By quenching the phase of the driving field, one can induce a dispersion relation featuring an energy crossing between an energetically stable charge mode and a dynamically stable spin mode. Quite generically, such a crossing becomes unstable when a perturbation is introduced to mix the modes. For our system, a finite detuning of the coupling-field frequency suffices. We propose an experimental protocol designed to test our result and predict the consequences of the instability.

This mechanism, which we term unstable avoided crossing, is a generic feature of systems with two degrees of freedom in a nonequilibrium state. We note that in traditional condensed-matter systems, negative frequencies are usually associated with dynamical instabilities (the Landau instability in superfluids, for instance). Our results can explain why, since in the presence of a dissipative bath, any negative-frequency mode can couple to a stable mode of the bath with the same (absolute) frequency and become unstable. In contrast, ultracold neutral atoms are extremely well insulated from the environment and negativefrequency modes are dynamically stable.

There are multiple pathways to extend this work. In particular, we assumed Bose-Einstein condensation, which does not occur in one-dimensional systems. One might study whether an avoided level crossing still arises for a quasicondensed Luttinger liquid. Furthermore, by adding an optical lattice, an effective momentum cutoff is introduced with the Brillouin zone. By varying the gas density, it 
should be possible to tune the cutoff and control the emergence of finite wave vector dynamical instabilities.

We would like to thank R. Barnett, I. Cirac, K. R. Islam, F. Mila, M. Oberthaler, and R. Schmidt for useful discussions. The authors acknowledge support from HarvardMIT CUA, the DARPA OLE Program, ARO-MURI on Atomtronics, and ARO-MURI Quism.

*nathan.bernier@alumni.epfl.ch

[1] M. C. Cross and P. C. Hohenberg, Rev. Mod. Phys. 65, 851 (1993).

[2] L. Santos, G. V. Shlyapnikov, and M. Lewenstein, Phys. Rev. Lett. 90, 250403 (2003).

[3] W. Zhang, D. L. Zhou, M.-S. Chang, M. S. Chapman, and L. You, Phys. Rev. Lett. 95, 180403 (2005).

[4] L. E. Sadler, J. M. Higbie, S. R. Leslie, M. Vengalattore, and D. M. Stamper-Kurn, Nature (London) 443, 312 (2006).

[5] R. M. W. van Bijnen, D. H. J. O’Dell, N. G. Parker, and A. M. Martin, Phys. Rev. Lett. 98, 150401 (2007).

[6] R. W. Cherng, V. Gritsev, D. M. Stamper-Kurn, and E. Demler, Phys. Rev. Lett. 100, 180404 (2008).

[7] A. Sartori and A. Recati, Eur. Phys. J. D 67, 260 (2013).

[8] R. Hipolito and A. Polkovnikov, Phys. Rev. A 81, 013621 (2010).

[9] J. Kronjäger, C. Becker, P. Soltan-Panahi, K. Bongs, and K. Sengstock, Phys. Rev. Lett. 105, 090402 (2010).

[10] E. Nicklas, H. Strobel, T. Zibold, C. Gross, B. A. Malomed, P. G. Kevrekidis, and M. K. Oberthaler, Phys. Rev. Lett. 107, 193001 (2011)

[11] P. Tommasini, E. J. V. de Passos, A. F. R. de Toledo Piza, M. S. Hussein, and E. Timmermans, Phys. Rev. A 67, 023606 (2003).

[12] The analogy between dynamically unstable modes and imaginary frequencies can be made explicit by the transformation $p^{\prime}=\sqrt{i} p, x^{\prime}=\sqrt{i} x$, satisfying $\omega\left(p^{2}-x^{2}\right)=$ $i \omega\left(x^{\prime 2}+p^{\prime 2}\right)$.

[13] R. Rossignoli and A. M. Kowalski, Phys. Rev. A 72, 032101 (2005).

[14] T.-L. Ho and V. B. Shenoy, Phys. Rev. Lett. 77, 3276 (1996).

[15] J. Stenger, S. Inouye, D. Stamper-Kurn, H.-J. Miesner, A. Chikkatur, and W. Ketterle, Nature (London) 396, 345 (1998).
[16] J. P. Burke, C. H. Greene, and J. L. Bohn, Phys. Rev. Lett. 81, 3355 (1998).

[17] C. Pethick and H. Smith, Bose-Einstein Condensation in Dilute Gases (Cambridge University Press, Cambridge, England, 2008).

[18] D. Hall, in Emergent Nonlinear Phenomena in Bose-Einstein Condensates, edited by P. Kevrekidis, D. Frantzeskakis, and R. Carretero-González, Atomic, Optical, and Plasma Physics Vol. 45 (Springer, Berlin, 2008), p. 307.

[19] B. Malomed, in Emergent Nonlinear Phenomena in Bose-Einstein Condensates, edited by P. Kevrekidis, D. Frantzeskakis, and R. Carretero-González, Atomic, Optical, and Plasma Physics Vol. 45 (Springer, Berlin, 2008), p. 287.

[20] We note that in the experiment, two-photon processes can be used, with the rf field combined with microwave radiation, for instance [10], with the same result.

[21] C. Chin, R. Grimm, P. Julienne, and E. Tiesinga, Rev. Mod. Phys. 82, 1225 (2010).

[22] S. B. Papp, J. M. Pino, and C. E. Wieman, Phys. Rev. Lett. 101, 040402 (2008).

[23] The population imbalance $f=\left(n_{1}-n_{2}\right) /\left(n_{1}+n_{2}\right)$ is given [11] by the solution of $\delta+\left(\lambda_{1}-\lambda_{2}\right) n+$ $\left(\frac{\lambda_{1}+\lambda_{2}}{2}-\lambda_{12}\right) n f+\frac{2 \alpha \times \cos \theta f}{\sqrt{1-f^{2}}}=0$, with $n=n_{1}+n_{2}$, and can have up to three possible values. In the symmetric case $\delta=0$ and $\lambda_{1}=\lambda_{2}$, one solution always corresponds to $f=0$. See also Tommasini et al. [11] for a discussion of the solutions around $\theta=0$ and the nature of the Bogoliubov expansion around it.

[24] L. Pitaevskii and S. Stringari, Bose-Einstein Condensation (Oxford University Press, New York, 2003).

[25] As can be seen by $x_{i}=\sqrt{2 n_{i}} \widehat{\delta \varphi}_{i}$ and $p_{i}=\widehat{\delta \rho}_{i} / \sqrt{2 n_{i}}$ that we obtain if we write $\hat{\psi}_{i}=e^{-i\left(\theta_{1}+\widehat{\delta \varphi}_{i}\right)}\left(n_{i}+\widehat{\delta \rho_{i}}\right)^{1 / 2}$ and expand it to first order.

[26] See Supplemental Material at http://link.aps.org/ supplemental/10.1103/PhysRevLett.113.065303, which includes Refs. [27,28], for more details about the experimental protocol.

[27] M. Vengalattore, S. R. Leslie, J. Guzman, and D. M. Stamper-Kurn, Phys. Rev. Lett. 100, 170403 (2008).

[28] S. Hofferberth, I. Lesanovsky, T. Schumm, A. Imambekov, V. Gritsev, E. Demler, and J. Schmiedmayer, Nat. Phys. 4, 489 (2008). 